\title{
Study on Application of Photoshop Software in the Interior Design Renderings
}

\author{
Liying WU \\ City Construction College of civil engineering \\ Jiujiang University \\ Jiujiang ,332000,China
}

\begin{abstract}
With the rapid development of computer technology, two-dimensional and three-dimensional technology continues is also attracting attention, computer graphics is more and more widely applied to various fields, especially with the popularity of decoration, interior design has become the gold industry, computer graphics has played an indispensable role in the interior design, has become an important means of expression of interior design the. Complete the design process: first using the Auto CAD idea sketch design, and then use 3Ds MAX to create the deployment model, import material, build cameras add light, render output, and finally the use of Photoshop post processing. Only through the Photoshop post processing to make the post effect diagram to achieve realistic results. This paper introduces some methods of Photoshop in interior design commonly used in making and adjustment.
\end{abstract}

Keywords-Mathematics; Interior design; Photoshop application; Common method; Effect of map processing;

\section{INTRODUCTION}

Post processing manufacture process in effect diagram can play a very important role. In contrast to the overall brightness, color of the renderings of the adjustment, which makes the effect of image become more really, natural. By adding a background character, plants, making the performance of rendering more angry, more expressive. The post processing software is the graphic image processing software Photoshop. In the late effect of image processing should add between background and 3D model scale, not too big, nor too small, also want to pay attention to the background and perspective of object perspective to be consistent. The projection position of the light, the shadow also should be unified, lighting effects to be consistent. Background not distracting, placement and size ratio in color, not too prominent, always let the scene in obedience to the Lord's status, the best foils.

In general we use Photoshop to change the tone, color renderings. Some in the rendering process will slow down the speed of the elements, many people will choose PS to post, such as some 3Ds Max no material, reflection, the brighter the tiles, there are some special circumstances, such as rendering device problems, or optical domain network, we can adjust the effect diagram using Photoshop shading and color.

\section{DEMAND ANALYSIS OF PHOTOSHOP PROCESSED IN INTERIOR RENDERINGS}

Designers use 2D and 3D etc effect chart manufacture software in interior renderings, and express their own creative design picture to the user with features of object modeling, structure, color, texture, and many other factors, make people more clearly understand the relationship between design of the performance, structure, materials, combining the method etc..

(1) The effect of the generated graph cannot meet the needs of designers

Image output from the rendering 3DS MAX, materials and lights always have unsatisfactory places, it needs to be adjusted in the late treatment process.

(2) The effect of the generated graph cannot meet the needs of users

Doing interior renderings before designers must do a lot of user needs analysis aspects of the work, but that time is an abstract stage, users can intuitively see the effect. So when the real generation effect diagram, the user must have this kind of demand. This stage designers need thorough analysis of the needs of users, the next step for renderings produced can allow the user to maximize satisfaction.

(3) Using Photoshop to deal with later time-saving convenience

If the adjustment of some materials and lighting such as the replacement of some object material, if adjusted in the 3DS MAX, then the Vray renderer to render, need a couple of hours or even a day. If the late treatment with Photoshop based on the original renderings, using minute can make we need effect. Some renderings we only images, not the 3D file original designers, not with the 3D software to adjust, only later were processed by Photoshop.

(4) The market currently cannot meet the demand of software

Home Furnishing magician software and siweixing decorative material software is currently on the market relatively well-known decorative material software, can use these software according to the objects of any user demand for replacement of material effect chart. But the picture effect generated light. Light information and original renderings difference is too large, in effect drawing generated relatively stiff, can't meet our needs. 


\section{LATER PROCESSING STEPS OF INTERIOR RENDERINGS}

(1) The observation and analysis of the picture

The late treatment before using Photoshop, must first find out the rendering problem, where only the question, to solve the problem very well. For example, a pair of rendering unsuccessful renderings, it has following several aspects. The whole image is too gray tone, the bright place no lights up, the dark place not go dark, did not reflect the changes in brightness. Scene modeling texture not well reflected, such as curtains, sofa and floor etc. the shape material. The light is too dim downlight, ceiling lamp with no production effect, appears to be relatively monotonous. The lack of character, plant landscape, the whole looks dull without vigor.

(2) To adjust the image overall effect

Rendering post-processing of first from the big start effect, grasp the overall relationship, so adjust the local effect (such as material and light adjustment) will not affect the results, and the local effect adjustment is also easy to master.

(3) To adjust the materials and lighting

Material and lighting affect each other. The material is mainly used to reflect the scene in the form of texture, lighting not only to illuminate the scene, can very good contrast scene atmosphere. Image output from the rendering 3DS MAX, materials and lights always have unsatisfactory places, it needs to be adjusted in the late treatment process.

(4) Produced the background

Background interior renderings include plants, people and some decorations etc.. The choice of material generally choose beautiful shape, color can be coordinated with the picture of the main color material, then according to the picture of the need for details on its adjustment.

\section{APPLICATION CASE OF PHOTOSHOP IN INTERIOR RENDERING POST-PROCESSING}

\section{A. According to user needs to adjust the material and light}

According to the needs of users, the designer produce effect diagram is shown in Figure 2, user mountain to a variety of reasons do not want to use the results in the figure of pale yellow carpet, and the switch to the side for the red carpet. The following is as an example that how to adjust the material in Photoshop and light.

(1) According to the needs of users, the need to use the original carpet selection tool after pull out in Photoshop, see figure 3 ;

(2) According to the needs of users, making for the aisle carpet laying;

(3) The produce aisle carpet laying to effect diagram in Figure 3, open, to make carpet using the move tool dragged into the figure 3 , the use of self-mountain deformation command roughly change carpet size, according to the perspective principle, hold down the Ctrl key, the four vertex to adjust the image's position, get the accord with three dimensional vision carpet laying effect, as shown in figure 4;

(4) Duplicate the background layer, the white area selection is to pull off the carpet with the magic wand tool, select the carpet layer, then use the anti-choice, delete redundant carpet, let the irregular background revealed, for example in the figure of the pillars etc.;
(5) The above steps have been completed the laying of new carpet general designer, this is the end of work. But in comparison to the original local lighting, carpet needs further processing;

(6) According to the original general adjust brightness and contrast images, and then observe the artwork found in this graph, the lights on the carpet is obvious. So according to the irradiated area lights, first using the selection tool select need to brighten areas. After selection of eclosion constituency constituency, make a smooth transition;

(7) Replication constituency, create a new layer and pasted constituency. Using the dodge tool to make selective brightening. The production of other places with the same method of lighting effects, and finally obtain the result in Figure 5.

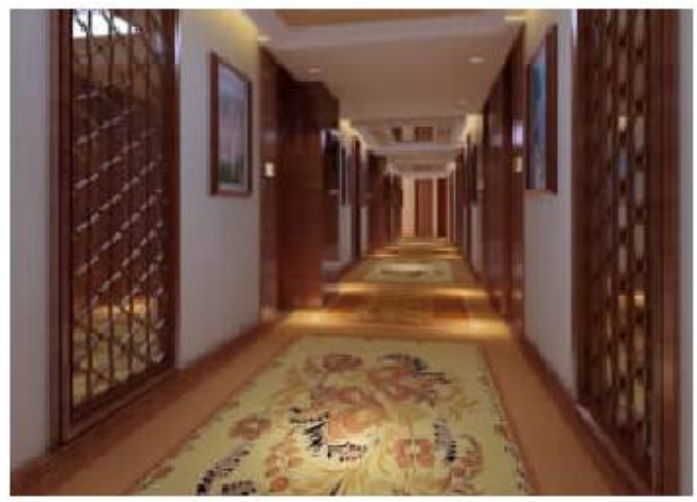

Fig.1 The original image

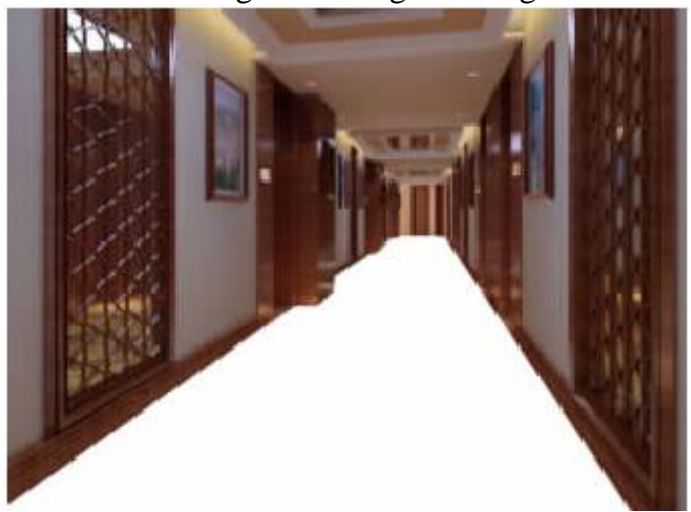

Fig.2 The image after matting

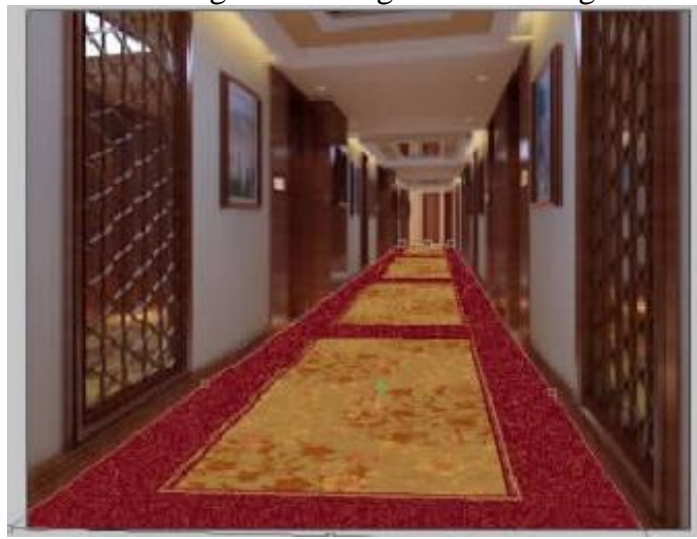

Fig.3 deformate and join the carpet image 


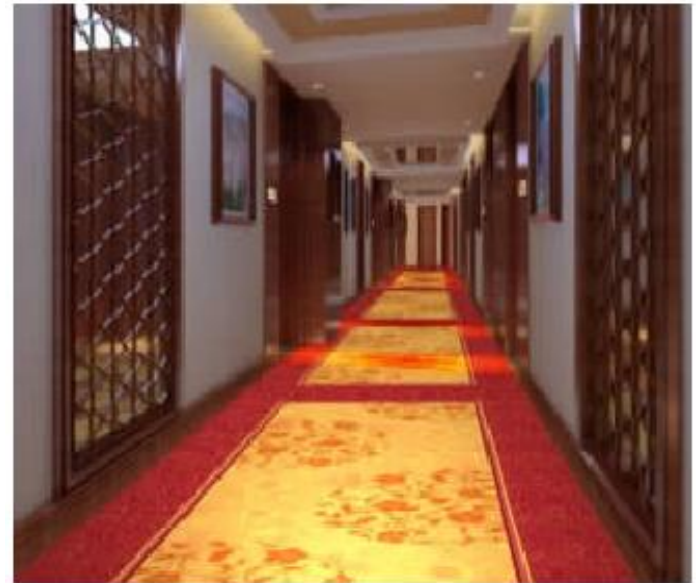

Fig.4 The image change the layer contrast

\section{B. "Replacement" filter change the material used to in the} indoor effect diagram

When the designer generate interior renderings show not to give users, the user will put forward many suggestions to the designers, which the user requires a change in material effect diagram is a regular thing. "Replacement" filter is especially suitable for the replacement of similar curtain has the effect of light and shade folds of material, the following to effect of pictures in the white curtain replacement for red patterned curtains as an example, Figure 6 is the original effect diagram, Figure 7 is the replacement generated renderings.

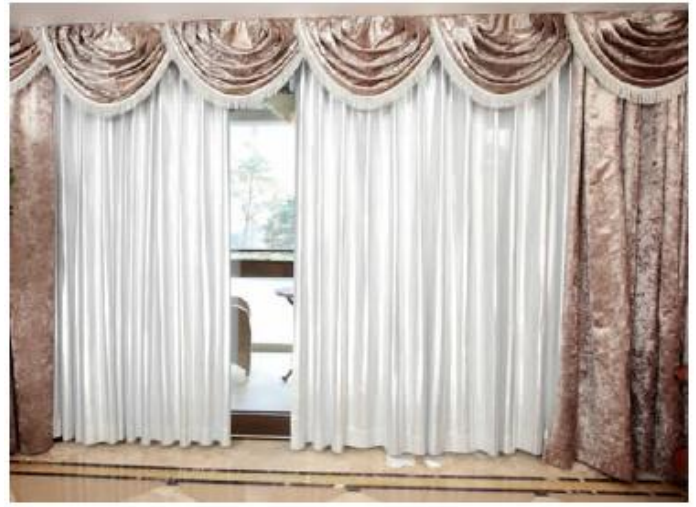

Fig.5 The original image

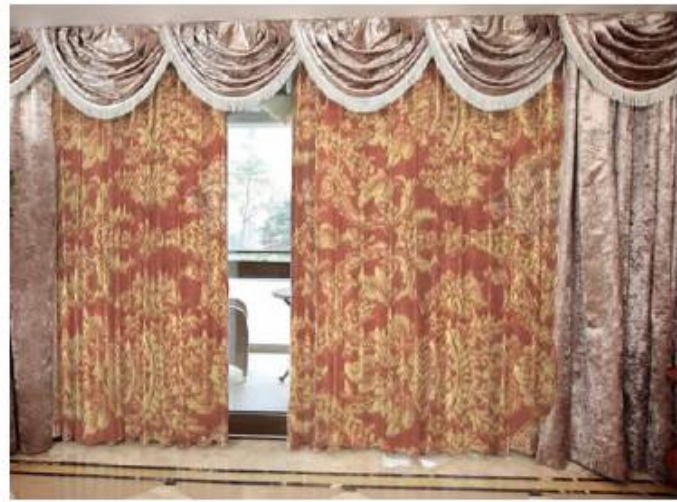

Fig.6 The image after replacement

\section{Using Photoshop channel to replace windows background}

(1) Open the image, as shown in figure 8.

(2) To observe the material, we can see buildings to formed side structure of the beauty of rhythm, but for the scenery outside the window is not satisfied, so want to replace the background. For such a complex background, obviously cannot use the magic wand tool or color range to create, this requires the use of channels to create constituencies.

(3) Transferred to need background image replacement, as shown in figure 9 .

(4) Create a constituency in the channel. Open the channels palette, select a larger contrast channel. Here is a selection of the blue channel, blue channel for copy. Select "image / high / curve" command, open the curve panel, the highest point of the curve and the lowest moved in to enlarge the picture contrast.

(5) Through the window to see some of the building at the airport, they are not necessary, select the brush tool in the toolbox, set the brush diameter and hardness appropriate. Here does not need the scenery to spread out with the brush, as shown in figure 10.

(6) Hold down the Ctrl key, use the mouse to click the current channel, see ants line in the image, load the channel selection, as shown in figure 11 .

(7) Replace the background in the layers panel. Click the RGB composite channel, see the color image. Click the layer panel, re open to replace the background layer in the layers palette, click and create a layer mask icon. You can see the current layer appeared on the layer mask, according to just loaded channels to create the layer mask just be part of the waiting hall window retention.

(8) The scenery outside the window has been replaced, the scenery is not satisfied can continue to adjust. Select the move tool in the toolbox, press the blue sky and white clouds moving images to the right place, the results are as shown in figure 12 .

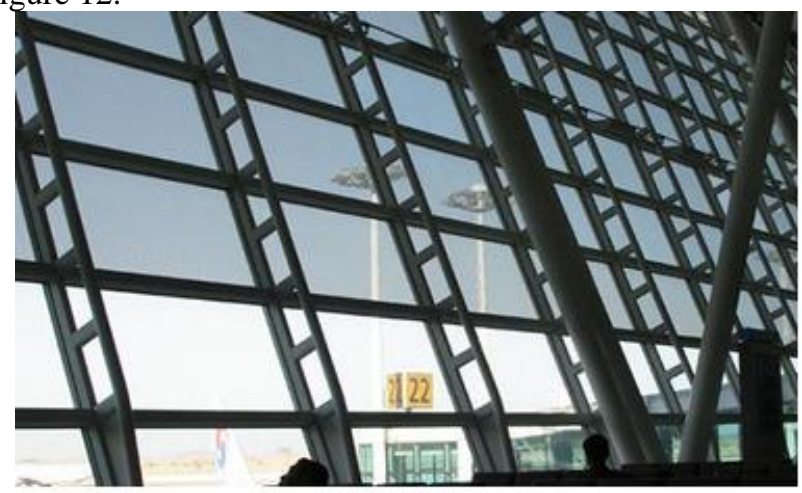

Fig.7 The original image 


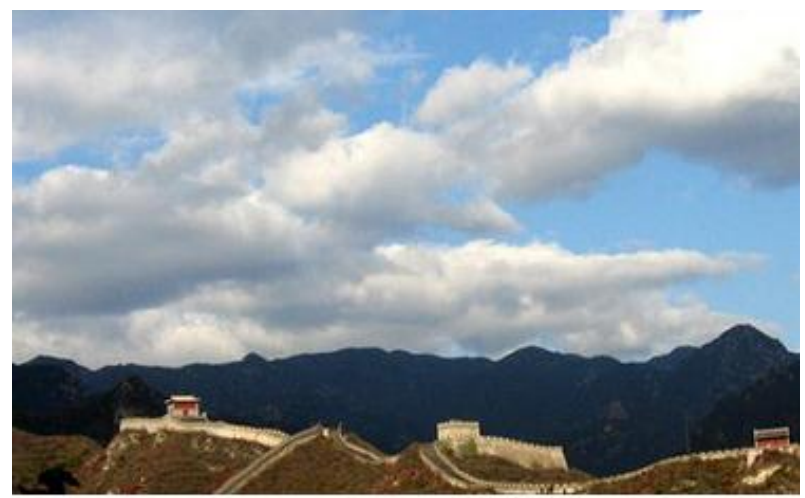

Fig.8 The background image material

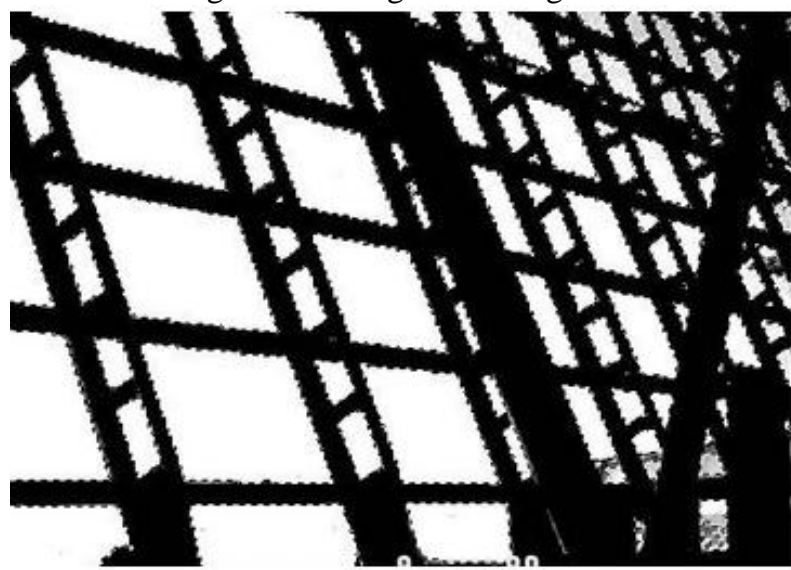

Fig.9 The blue channel image

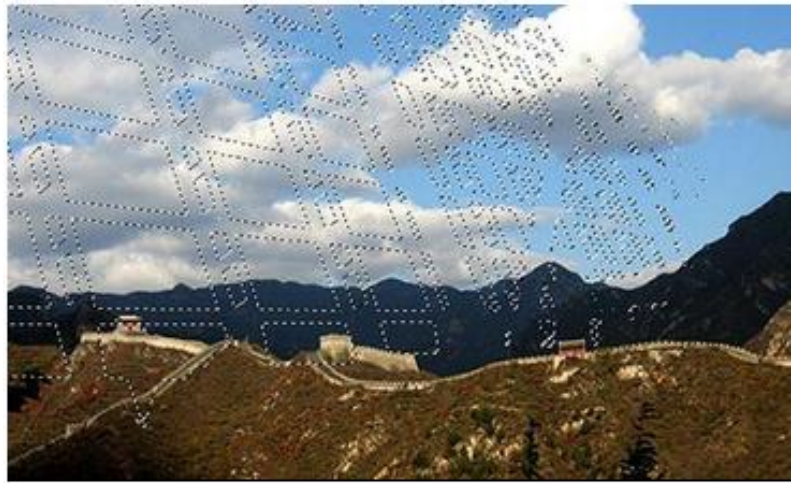

Fig.10 Load the ants selection lines

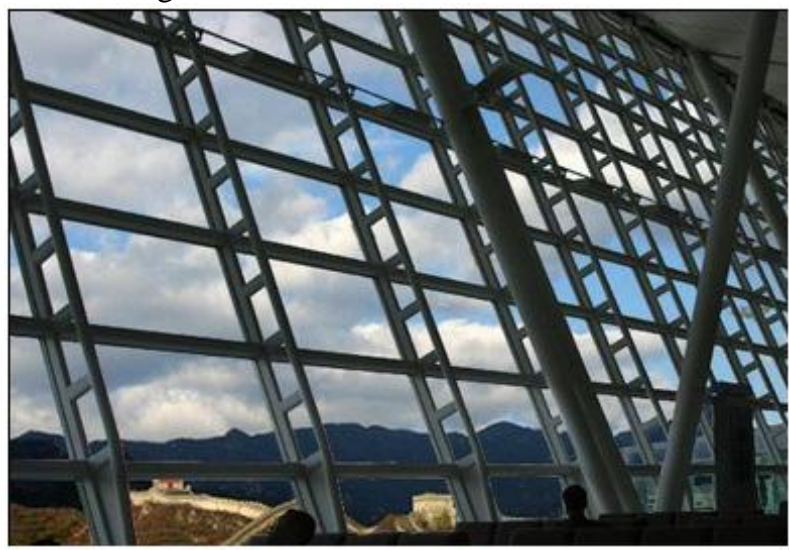

Fig.11 The final completion image

\section{CONCLUSIONS}

Photoshop will bring many convenience for designers in interior rendering post-processing, save a lot of time. Especially for the adjustment of the material and the light is incomparable with other software, using Photoshop can quickly produce different effects in different materials, different lighting effects create shading objects locally, create objects in the sun or light projection effect. So correctly and skillfully master the methods and techniques in the interior renderings later processing in Photoshop is the ability of each interior designer must have.

\section{REFERENCE}

[1] Song Aihui. Research on the use of modern information technology in the teaching Art [J]. knowledge economy, 2013 (14) $165-176$

[2] Ji shu. The leading creative thinking of in the computer arts design [J]. Education and innovation, 2012 (2): 32

[3] Song fan. Research on the relationship between computer art and art [J]. China Chapter, 2012 (13): 71

[4] Niu Guoqiang, Discuss on the application of computer technology in art and design [J]. Education Teaching Forum, 2014 (5): 248

[5] Wang Fanglin, The basic art theory in the computer arts design [J] Chinese teachers, 2013 (3): 51-52

[6] Wang Li. mathematics colleges teaching the new curriculum ideas [J]. Adult Education,2011,09:108 -109.

[7] Chen $\mathrm{Yu}$, Zheng Li. Innovation Research and Teaching Mathematics Teaching System Model vocational [J]. Technological information,2011,33:146 -147.

[8] Orihong. Improving vocational colleges teaching mathematics to explore the effect of $[\mathrm{J}]$. Higher Education Forum,2005,01:155 158 .

[9] Shi Xiaojun. Mathematics curriculum and vocational colleges teaching mode to optimize discuss reform [J]. Era of education ( education edition ), 2009,10:38.

[10] Cao Yuping. Mathematics vocational colleges teaching quality evaluation system [J]. Education Forum,2013,11:35 -38.

[11] Fan Xiaohui. outstanding problems in mathematics teaching vocational colleges and countermeasures. [J]. Technological innovation Herald, 2013,07:178.

[12] Shi Junying. experimental vocational colleges teaching mathematics research [D]. Shandong Normal University, 2011.

[13] Xu Ying. Mathematics Teaching Model of innovation and reform vocational colleges [J]. Chinese EDUCATION INNOVATION HERALD,2014,05:20 -21.

[14] Xue Xuejun analysis and thinking Mathematics teaching status of vocational colleges [J]. Education and Vocational,2007,21:111 112.

[15] Lixin Ke. Several Mathematics Teaching in Vocational Institutions [J]. Zhejiang Youth College,2006,01:21 -23 